Fisioter Bras 2018;19(1):1-2

\title{
EDITORIAL \\ Exercício terapêutico na esclerose lateral amiotrófica: o que esperamos da relação anabolismo vs. catabolismo?
}

Marco Orsini ${ }^{*}$, Mauricio de Sant' Anna $\mathrm{Jr}^{\star *}$, Marcos RG de Freitas ${ }^{\star * *}$, Marco Felipe Bouzada*, Manuel Leite Lopes ${ }^{\star \star * \star}$, Acary Bulle Oliveira****

*Programa de Mestrado e Doutorado em Ciencias da Reabilitação - UNISUAM, RJ, Programa de Mestrado em Ciências Aplicadas à Saúde, Universidade Severino Sombra, **Programa de Mestrado em Ciências Aplicadas à Saúde, Universidade Severino Sombra, ${ }^{* * *}$ Instituto Federal do Rio de Janeiro, IFRJ, Curso de Fisioterapia, ${ }^{* * * *}$ Clinica Sinapse, Rio de Janeiro/RJ, *****Escola Paulista de Medicina, Universidade Federal de São Paulo, UNIFESP

Correspondência: Marco Orsini, E-mail: orsinimarco@hotmail.com

A esclerose lateral amiotrófica (ELA) pode ser definida como uma doença neurológica progressiva, degenerativa e inexorável, cuja gênese ainda é de difícil entendimento. Fatores vários contribuem na desprogramação e morte celular precoces. A busca por uma melhor interrelação, em novos marcadores e associações com tipos celulares elou moleculares distintos parece $o$ grande desafio. $O$ delineamento de pesquisas clinicas em (ELA), com informações a respeito de tipo, ação e dose de medicamentos emergem à medida que novas teorias são apresentadas e somadas ao modelo atual $[1,2]$.

Definitivamente a ELA é uma doença multifatorial. $O$ estresse oxidativo, excitotoxicidade mediada pelo glutamato, efeitos causados pela mutação do superóxido dismutase, agregação anormal proteíco-especifica, desestruturação de neuro-filamentos intermediários, alteração do transporte axonal anterógrado e retrógrado, ativação microglial, inflamação e transtornos nos fatores de crescimento, têm sido considerados como potenciais agressores aos neurônios motores. Fatores genéticos, influxo excessivo de cálcio intracelular e apoptose também fazem parte desse compreensível mas ainda indecifrável modelo teórico [3].

Baseado no exposto sobre a fisiopatologia da ELA, bem como sobre as manifestações clínicas dessa doença, torna-se evidente a necessidade de atuação multidisciplinar, em especial do fisioterapeuta desde o momento do diagnóstico clínico, e que será o responsável pela prescrição dos exercícios terapêuticos.

Mas o que esperamos da relação anabolismo vs. catabolismo, uma vez que desde a fase inicial da ELA preconiza-se a realização de exercício terapêuticos das mais diversas naturezas (aeróbicos, resistido, respiratórios etc), porém a cadeia de eventos devastadores inerentes à fisiopatologia da ELA já estão em franca evolução? Respeitando tanto os objetivos terapêuticos como as fases da ELA, a prescrição do fortalecimento muscular e 0 recondicionamento físico tornam-se componentes do programa de tratamento estabelecido pela da fisioterapia, e diversos estudos são encontrados na literatura, e que sustentam sua prática, porém com resultados controversos [4-6].

Podemos dividir o gasto energético diário em três componentes: taxa metabólica basal, efeito térmico do alimento e gasto energético associado com a atividade física. A realização de um exercício físico promove aumento do gasto energético total, tanto aguda, quanto cronicamente. No que tange o efeito agudo, encontra-se bem estabelecido que após o término da atividade, o consumo de oxigênio $\left(\mathrm{VO}_{2}\right)$ não retorna aos valores de repouso, imediatamente. Essa demanda energética durante o período de recuperação após o exercício, que é necessária para "quitar a divida metabólica assumida durante a realização da atividade" é definida como excess post-exercise oxygen consumption ou consumo excessivo de oxigênio após o exercício (EPOC) [7,8]. Em virtude do grande desajuste metabólico que envolve a ELA, e do franco estado catabólico, o questionamento que deve ser feito é, se a intensidade do exercício prescrito não irá gerar um efeito EPOC, que pode vir a ser prejudicial a esse grupo de pacientes? Seria então essa "dívida metabólica" mais um trabalho a ser executado por um organismo deveras debilitado e em franco catabolismo? Existem novas possibilidades terapêuticas num arcabouço fisiopatológico ainda em construção, uma delas é a proposta do protocolo Deanna [9] que sugere a suplementação de arginina-alfa-cetoglurarato, complexo B, dentre outras poderiam atenuar a disfunção mitocondrial, excitotoxicidade do glutamato e estresse oxidativo. É fundamental que a prescrição do exercício seja em intensidade 
submáxima (40 -60\% $\mathrm{VO}_{2}$ pico, ou $60-85 \%$ FCmáx ou $10-13$ na escala de Borg) tanto pelo aspecto que envolve o efeito EPOC, assim como pelos efeitos diretamente relacionados ao metaborreflexo da musculatura periférica e respiratória. Pautados nesses conceitos não contra indicamos a prescrição do fortalecimento muscular e o recondicionamento físico e portadores de ELA, porém sugerimos que todos esses aspectos sejam contemplados na prescrição.

\section{Referencias}

1. Orsini M, Oliveira AB, Nascimento OJM, Reis CHM, Leite MAA, Souza JA, Pupe C, Souza OG, Bastos VH, Freitas MRG, Teixeira S, Bruno C, Davidovich E, Smidt B. Amyotrophic lateral sclerosis: new perpectives and update. Neurol Int 2015;7(2):5885. doi: 10.4081/ni.2015.5885.

2. Chieia MA, Oliveira ASB, Silva HCA, Gabbai AA. Considerations on diagnostic criteria. Amyotrophic lateral sclerosis. Arq Neuropsiquiatr 2010;68(6):837-842

3. Oliveira AS, Pereira RD. Amyotrophic lateral sclerosis (ALS): three letters that change the people's life for ever. Arq Neuropsiquiatr 2009;67(3A):750-82. doi:10.1590/S0004282X2009000400040.

4. Drory VE, Goltsman E, Reznik JG, Mosek A, Korczyn AD. The value of muscle exercise in patients with amyotrophic lateral sclerosis. J Neurol Sci 2001; 15;191(1-2):133-7. doi10.1016/j.pmr.2008.02.003

5. Dalbello-Haas V, Florence JM, Krivickas LS. Therapeutic exercise for people with amyotrophic lateral sclerosis or motor neuron disease. Cochrane Darabase Syst Rev 2008;16;(2):CD005229. doi: 10.1002/14651858.CD005229.pub2.

6. Chen $\mathrm{A}$, Montes $\mathrm{J}$, Mitsumoto $\mathrm{H}$. The role of exercise in amyotrophic lateral sclerosis. Phys Med Rehabil Clin N Am 2008;19(3):545-57, ix-x. doi: 10.1016/j.pmr.2008.02.003.

7. Sedlock DA, Lee MG, Flynn MG, Kamimori GH. Excess postexercise oxygen consumption after aerobic exercise training. Int J Sport Nutr Exerc Metab 2010;20(4):336-49.

8. Tucker WJ, Angadi SS, Gaesser GA. Excess post-exercise oxygen consumption after high-intensity and sprint interval exercise, and continuous steady-state exercise. J Strength Cond Res 2016;30(11):3090-3097.

9. ALSUntangled Group, Fournier C, Bedlak B, Hardiman O, Heiman-Patterson T, Gutmann L et al. ALSUntangled Group. ALS Untangled No. 20: The Deanna Protocol: Amyotrophic Lateral Scler Frontotemporal Degener. 2013;14(4):319-23.

10. Dempsey JA, Romer L, Rodman J, Miller J, Smith C. Consequences of exerciseinduced respiratory muscle work. Respir Physiol Neurobiol 2006;28;151(2-3):242-50. 